\title{
ON MINIMAL SETS IN THE PLANE
}

\author{
RUSSELL REMAGE, JR. ${ }^{1}$
}

This paper gives by example an affirmative answer to two questions raised by M. L. Cartwright (personal communication). See [1] The first is based on an example by F. B. Jones of a minimal orbitclosure in the plane which is locally connected at some points and not at others (see [2, pp. 139-142]), and asks whether there is an orientation-preserving homeomorphism of the plane onto itself which has this set as a minimal orbit-closure. The second may be phrased "Does there exist an orientation-preserving homeomorphism of the plane onto itself which admits a minimal orbit-closure with an infinite number of nondegenerate components?". In the first of the examples there is also an answer, in the negative, to that part of the question of Bass, as quoted in [3], which asks whether a minimal orbit-closure of dimension $n-1$ on an $n$-dimensional manifold is necessarily an $(n-1)$-torus.

We adopt, generally, the notation of [2]. Let $x$ be used to denote a variable on $R$, the real line, and $\theta$ a variable on the unit circle $\mathcal{C}$, each with its natural topology. Let $\omega$ be the mapping of $\mathbb{R}$ onto $\mathfrak{C}$, defined by $\omega(x)=(1, x)$ where the right-hand side is in polar coordinates $r$ and $\theta$. Normally, barred symbols will be used when $\mathfrak{C}$ is involved, unbarred when $R$ is under consideration. That is, $f(x)$ is a function on $R ; \bar{f}(\theta)$ has domain $\mathcal{C}$.

Let $x_{0}$ be a real number, and let $f_{0}(x)$ be a real function continuous on $\left[x_{0}-\pi, x_{0}+\pi\right]$ except at $x_{0}$, periodic of period $2 \pi$, with $f_{0}\left(x_{0}-\pi\right)$ $=f_{0}\left(x_{0}+\pi\right)$, and satisfying the inequality $\left|f_{0}(x)\right| \leqq 1$. Let $\tau$ be a homeomorphism of $R$ onto itself, such that $\tau(x+2 \pi)=\tau(x)+2 \pi$. If $g$ is the set of all integers, let $X_{n}=R-\left\{\tau^{n}\left(x_{0}\right)+2 k \pi / k \in g\right\}$, $X=\bigcap_{-\infty}^{\infty} X_{n}$.

The relation $f_{n}(x)=f_{n+1}(\tau(x)), x \in X_{n}$, serves to define $f_{n}(x)$ for each $n \in g$. Let $\left(\alpha_{n}\right)$ be a bisequence of constants, positive for convenience, such that $\sum_{n=-\infty}^{\infty} \alpha_{n}$ converges, so that $\sum_{-\infty}^{\infty} \alpha_{n} f_{n}(x)$ converges uniformly and absolutely. Let $\sum_{-\infty}^{\infty} \alpha_{n}=A, f(x)=2 A$ $+\sum_{-\infty}^{\infty} \alpha_{n} f_{n}(x)$.

Theorem 1. If $N_{1}=\{(x, f(x)) / x \in X\}$ and $\psi(x, f(x))=(\tau(x) . f(\tau(x)))$,

Presented to the Society, April 8, 1961 ; received by the editors November 4, 1960.

1 This paper was written while the author was a National Science Foundation Faculty Fellow. 
then $\psi$ and $\psi^{-1}$ are uniformly continuous homeomorphisms of the subset $N_{1}$ of the plane onto $N_{1}$.

The case in which $\tau$ is a translation by one unit is due to $F$. B. Jones. An account may be found on pp. 140-141 of [2]. Theorem 1 follows from Theorem 2 by restricting attention to $a=1$.

For $n \in g, 0 \leqq a \leqq 1$, and $\tau^{-n}(x) \in X_{0} \cap\left[x_{0}-\pi, x_{0}+\pi\right]$, let

$$
\begin{aligned}
e(x, n)= & \frac{\left|\tau^{-n}(x)-x_{0}\right|}{2 \pi} \\
g_{n}(x, a)= & a\left[2+f_{n}(x)\right]\left[1-(1-a)^{\bullet(x, n)}\right] \\
& +a(1-a)^{\bullet(x, n)}
\end{aligned}
$$

for $n \in g, 1 \leqq a \leqq 2$, and $\tau^{-n}(x) \in X_{0} \cap\left[x_{0}-\pi, x_{0}+\pi\right]$, let

$$
\begin{gathered}
g_{n}(x, a)=a\left[2+f_{n}(x)\right][1-(a-1) \cdot(x, n) \\
+(2+a)(a-1) \bullet(x, n)
\end{gathered}
$$

Let the domain of $g_{n}(x, a)$ be extended by defining $g_{n}(x, a)$ to be periodic of period $2 \pi ; g_{n}(x+2 \pi, a)=g_{n}(x, a), x \in X_{n}, 0 \leqq a \leqq 2$.

For $x \in X$, let $g(x, a)=\sum_{-\infty}^{\infty} \alpha_{n} g_{n}(x, a)$, and let $\Psi(x, g(x, a))$ $=(\tau(x), g(\tau(x), a))$.

TheOREM 2. $\Psi$ and $\Psi^{-1}$ are uniformly continuous homeomorphisms of $X \times[0,4 A]$ onto itself.

Proof. We first make some observations about $g_{n}(x, a)$ and $g(x, a)$. For $x \in X_{n}$,

$$
\begin{aligned}
& g_{n}(x, 0)=0, \quad g_{n}(x, 1)=2+f_{n}(x), \quad g_{n}(x, 2)=4, \\
& \lim _{x \rightarrow \tau^{n}\left(x_{0}\right)} g_{n}(x, a)=\left\{\begin{array}{cc}
a, & 0 \leqq a \leqq 1 \\
2+a, & 1 \leqq a \leqq 2 .
\end{array}\right.
\end{aligned}
$$

For $x \in X$,

(c) $g(x, 0)=0, g(x, 1)=f(x), g(x, 2)=4 A$.

(d) For $0 \leqq a \leqq 2, a \neq 1, \partial g_{n}(x, a) / \partial a>0$, so that $g(x, a)$ is a strictly increasing function of $a$.

(e) $g(x, a)$ is a continuous function of $x$ and $a$ on $X \times[0,2]$; and for each $x \in X, g(x, a)$ maps an ordinate set $\{(x, a) / 0 \leqq a \leqq 2\}$ one-toone onto an ordinate set $\{(x, y) / 0 \leqq y \leqq 4 A\}$.

(f) If $a \neq 1$ is fixed, from (b), $g_{n}(x, a)$, as a function of $x$, has a continuous extension to $R$, as does $g(x, a)$.

(g) $g_{n}(\tau(x), a)=g_{n-1}(x, a), 0 \leqq a \leqq 2$. 
It is immediately clear that $\Psi$ is continuous on $X \times[0,4 A]$ and onto $X \times[0,4 A]$. We now show that $\Psi$ is uniformly continuous, following the line of the argument on pp. 140-141 of [2].

If $\Psi$ is not uniformly continuous, there is an $\epsilon>0$ such that for every $\delta>0$, there are points $(x, g(x, a))$ and $\left(x^{\prime}, g\left(x^{\prime}, a^{\prime}\right)\right)$ such that

$$
\begin{gathered}
\left|x-x^{\prime}\right|<\delta \text { and }\left|g(x, a)-g\left(x^{\prime}, a^{\prime}\right)\right|<\delta, \\
{\left[\left(\tau(x)-\tau\left(x^{\prime}\right)\right)^{2}+g(\tau(x), a)-\left(g\left(\tau\left(x^{\prime}\right), a^{\prime}\right)\right)^{2}\right]^{1 / 2}>2 \epsilon .}
\end{gathered}
$$

We may assume $\delta$ so small that, on account of the uniform continuity of $\tau,\left|\tau(x)-\tau\left(x^{\prime}\right)\right|<\epsilon$, so that

$$
\left|g(\tau(x), a)-g\left(\tau\left(x^{\prime}\right), a^{\prime}\right)\right|>\epsilon .
$$

Let $\left(\delta_{n}\right)$ be a sequence of positive numbers tending steadily to zero; and $x_{n}, x_{n}^{\prime}, a_{n}, a_{n}^{\prime}$ numbers so that, for each $n>0$, (1) and (3) are satisfied. We may assume convergence:

$$
\lim x_{n}=x^{*}=\lim x_{n}^{\prime}, \quad \lim a_{n}=a^{*}=\lim a_{n}^{\prime} .
$$

If $a^{*} \neq 1$, say $a^{*}<1$, we may assume that $\sup _{n>0}\left(a_{n}, a_{n}^{\prime}\right)=b^{*}<1$. By observation (b), we can extend $g(x, a)$ on $X \times\left[0, b^{*}\right]$ to a periodic function $g(x, a)$, defined on $R \times\left[0, b^{*}\right]$. Since $\left[-\pi+x_{0}, \pi+x_{0}\right]$ $\times\left[0, b^{*}\right]$ is compact, $g(x, a)$ is uniformly continuous; hence so also is $g(x, a)$ on $X \times\left[0, b^{*}\right]$. This contradicts (1) and (3) for the sequences $\left(\delta_{n}\right),\left(a_{n}\right),\left(a_{n}^{\prime}\right),\left(x_{n}\right),\left(x_{n}^{\prime}\right)$.

We may now assume that $a^{*}=1$; further, on account of the continuity of $\Psi$ on $X \times[0,4 A]$, we may assume that $x^{*} \notin X$; there exist, then, integers $k$ and $n$ such that $x^{*}=2 k \pi+\tau^{m-1}\left(x_{0}\right)$, and on account of the periodicity we may take $x^{*}=\tau^{m-1}\left(x_{0}\right)$.

Since the series $\sum_{-\infty}^{\infty} \alpha_{n} g_{n}(x, a)$ is uniformly bounded, we can find a positive integer $P$ such that

$$
\sum_{n=P} \alpha_{n}\left(g\left(\tau\left(x_{k}^{\prime}\right), a_{k}^{\prime}\right)-g_{n}\left(\tau\left(x_{k}\right), a_{k}\right)\right) \mid<\epsilon / 3
$$

and similarly for the range $-\infty$ to $-P$. Since for $-P \leqq n \leqq P$, $n \neq m-1, g_{n}(\tau(x), a)$ is continuous at $\left(x^{*}, a^{*}\right)$, for sufficiently large $k$,

$$
\begin{aligned}
& \alpha_{n}\left|g_{n}\left(\tau\left(x_{k}^{\prime}\right), a_{k}^{\prime}\right)-g_{n}\left(\tau\left(x_{k}\right), a_{k}\right)\right|<\epsilon / 12 P \text { by }(1), \\
& \alpha_{m-1}\left|g_{m-1}\left(x_{k}^{\prime}, a_{k}^{\prime}\right)-g_{n}\left(x_{k}, a_{k}\right)\right| \\
& \leqq \delta_{k}+\left|\sum_{-\infty}^{-N} \alpha_{n}\left(g\left(x_{k}^{\prime}, a_{k}^{\prime}\right)-g_{n}\left(x_{k}, a_{k}\right)\right)\right| \\
& \quad+\left|\sum_{N}^{\infty} \alpha_{n}\left(g_{n}\left(x_{k}^{\prime}, a_{k}^{\prime}\right)-g_{n}\left(x_{k}, a_{k}\right)\right)\right|+\sum_{-N}^{N} \alpha_{n} \mid g_{n}\left(x_{k}^{\prime}, a_{k}^{\prime}\right)-g_{n}\left(x_{k}, a_{k}\right) .
\end{aligned}
$$


If $N$ is sufficiently large, the last of these can be made less than, say $\left(\alpha_{m-1} / \alpha_{m}\right)(\epsilon / 36)$ for all large $k$; if $k$ is sufficiently large the two preceding sums are also less than $\left(\alpha_{m-1} / \alpha_{m}\right)(\epsilon / 36)$, and also $\delta<\left(\alpha_{m-1} / \alpha_{m}\right)(\epsilon / 36)$. We thus have, for $k>k_{0}$,

$$
\alpha_{m-1}\left|g_{m-1}\left(x_{k}^{\prime}, a_{k}^{\prime}\right)-g_{m-1}\left(x_{k}, a_{k}\right)\right|<\left(\alpha_{m-1} / \alpha_{m}\right)(\epsilon / 9) .
$$

By $(g)$, however, $g_{m-1}(x, a)=g_{m}(\tau(x), a)$, so that

$$
\alpha_{m}\left|g_{m}\left(\tau\left(x_{k}^{\prime}\right), a_{k}^{\prime}\right)-g_{m}\left(\tau\left(x_{k}\right), a_{k}\right)\right|<\epsilon / 9 .
$$

Combining inequalities (4), (5), and (6), we have, for sufficiently large $k,\left|g\left(\tau\left(x_{k}^{\prime}\right), a_{k}^{\prime}\right)-g\left(\tau\left(x_{k}\right), a_{k}\right)\right|<\epsilon$ contradicting (3). This establishes the uniform continuity of $\Psi$, and by the symmetry of the $\tau, \tau^{-1}$ relation, also of $\Psi^{-1}$.

It follows that $\Psi$ and $\Psi^{-1}$ both have unique continuous extensions, $\hat{\Psi}$ and $\hat{\Psi}^{-1}$ to $R \times[0,4 A]$, and that both are homeomorphisms.

We now turn to consideration of the unit circle $\mathcal{C}$ in place of $R$, so that $\tau(x)$ is replaced by $\bar{\tau}(\theta)=\omega \tau \omega^{-1}, \hat{\Psi}$ by $\bar{\Psi}$ defined by $\bar{\Psi}(\theta, g(\theta, a))$ $=(\bar{\tau}(\theta), g(\bar{\tau}(\theta), a))$, for $\theta \in \Theta$, extending to a homeomorphism of the cylinder $\mathcal{C} \times[0,4 A]$ onto itself. Let $\Theta_{n}=\omega\left(X_{n}\right), \Theta=\omega(X), \theta_{0}=\omega\left(x_{0}\right)$. On the cylinder, let $C(a)$ be the closure of the set $\{(\theta, \bar{g}(\theta, a)) / \theta \in \Theta\}$, and denote by $\bar{\psi}_{a}$ the restriction of $\bar{\Psi}$ to $C(a)$. Formally, we state

CoROllaRy 1. $\bar{\Psi}$ is a homeomorphism of the cylinder $\mathfrak{e} \times[0,4 A]$ onto itself, such that each of the sets $C(a)$. for $0 \leqq a \leqq 2$ is invariant.

We note that, if $a \neq 1$, the projection $\pi$ of $C(a)$ onto $\mathfrak{C}$, defined by $\pi(\theta, \bar{g}(\theta, a))=\theta$, is by observation (f) one-to-one, and thus a homeomorphism, so that $C(a), a \neq 1$, is a simple closed curve.

If $\sigma$ denotes the "stereographic" projection of the cylinder $\mathcal{E} \times[0,4 A]$ onto the compact complex plane $\mathcal{P}$, so that the points of $\mathcal{C} \times\{0\}$ go to the origin $0, \mathcal{e} \times\{2 A\}$ to the unit circle, $\mathcal{e} \times\{4 A\}$ to the point at infinity, then $\sigma$, which is a homeomorphism on $\mathfrak{e} \times\{0,4 A\}-\{\mathfrak{e} \times\{0\} \cup \mathfrak{C} \times\{4 A\}\}$, induces a homeomorphism

$$
\begin{aligned}
\bar{\Phi} & =\sigma \Psi \sigma^{-1} \text { on } \beta-\{0\}-\{\infty\}, \\
\bar{\Phi}(0) & =0 .
\end{aligned}
$$

Clearly $\bar{\Phi}$ is orientation-preserving. Thus

COROLlARY 2. $\bar{\Phi}$ is an orientation-preserving homeomorphism of the plane $P$ onto itself, with the origin fixed, such that the origin, the set $C(1)$, and a continuous collection of simple closed curves filling up $\rho-\{0 \cup \sigma e(1)\}$ are invariant. 
Similar remarks, of course, can be made for the Riemann sphere instead of $P$.

Two examples utilizing these results follow. Although the functions given are specific, it is clear that many others would serve as well.

Example 1. (Transitive Case). In $[-\pi, 0)$ and $(0, \pi]$, let $f_{0}(x)$ $=\sin \pi^{2} / x$, so that $x_{0}=0$, let $f_{0}(x)$ be periodic of period $2 \pi$, and let $\bar{\tau}$ be a rotation of $\mathcal{e}$ by an irrational multiple $m$ of $2 \pi$, so that $\bar{\tau}(\theta)$ $=\theta+m$. Clearly $C(a)$ is a minimal orbit-closure for each $a$, and $\bar{\Psi}$ is pointwise almost periodic, almost periodic on $\mathcal{e} \times[0,4 A]-C(1)$; $C(1)$ is Jones' set, locally connected at some points but not at others. The same remarks hold for $\bar{\Phi}$, furnishing an example of an orbitclosure of dimension one on a two-dimensional manifold which is not a torus.

Modifying slightly the notation, it is easy to make an extension to $n$ dimensions: If $S^{n}$ denotes the $n$-sphere, let $\bar{\Phi}$ be considered as acting on $S^{2}$, and let $n$ be greater than two. Let $\bar{\tau}_{j}, j=1,2, \cdots, n-2$ be homeomorphisms of $S^{1}$ onto itself. Then, in general, $\bar{\Phi}^{n}\left(p ; \theta_{1}, \theta_{2}, \cdots, \theta_{n-2}\right)=\left(\bar{\Phi}(p) ; \tau_{1}\left(\theta_{1}\right), \cdots, \tau_{n-2}\left(\theta_{n-2}\right)\right)$ is a homeomorphism of the $n$-manifold $\mathfrak{N}^{\prime}=S^{2} \times S^{1} \times S^{1} \times \cdots \times S^{1}=S^{2} \times \mathbb{N}^{n-2}$ onto itself such that each set $C(a) \times \Re^{n-2}$ is invariant. In particular, if $\bar{\tau}, \bar{\tau}_{1}, \bar{\tau}_{2}, \cdots, \bar{\tau}_{n-2}$ are rotations of $S^{1}$ about the origin through angles $\theta, \theta_{1}, \theta_{2}, \cdots, \theta_{n-2}$ such that no linear combination of $\theta, \theta_{1}, \cdots$, $\theta_{n-2}$ and $2 \pi$ with integral coefficients vanishes, then clearly $C(a)$ $\times \mathbb{N}^{n-2}$ is a minimal set, so that $C(1) \times \Re^{n-2}$, although of dimension $n-1$, is not an $(n-1)$-torus.

Example II. (Intransitive Case). Let $H$ be the Cantor ternary set on $[0,2 \pi]$, with $(2 \pi / 3,4 \pi / 3)$ the largest complementary middle third, and let $K=\omega(H)$ be the corresponding set on the unit circle $\mathfrak{e}$. Let $\rho$ be a rotation of some (other) unit circle by an irrational multiple $m$ of $2 \pi$, and let $x$ be a definite point of this circle. Beginning with $I_{0}$, the closure of the arc $(2 \pi / 3,4 \pi / 3)$ complementary to $K$, arrange the closures of the arcs complementary to $K$ in a bisequence to have the same cyclic order as the bisequence $\left(\rho^{n}(x) / n \in g\right)$, choosing correspondents $I_{n}$ to $x_{n}$ in the order $I_{0}, I_{1}, I_{-1}, I_{2}, I_{-2}, \cdots$, by choosing the largest arc available to maintain the proper cyclic order with the arcs already chosen. Clearly the choice is unique after $I_{1}$ has been chosen. Let $\bar{\tau}$ be the homeomorphism of the circle $\mathcal{C}$ onto itself such that for each $n, \bar{\tau}\left(I_{n}\right)=I_{n+1}$. It is known (see [3]) that $K$ is a minimal set under $\bar{\tau}$, so that any definite point $\theta_{0} \in K$ is an almost periodic point under $\bar{\tau}$, and, if $T(x)$ denotes the orbit of $x, \mathrm{Cl}\left[T\left(\theta_{0}\right)\right]=K$. It follows that there is a relatively dense subset $\left\{n_{k} / k \in g\right\}$ of $g$ such that $\lim _{k \rightarrow \infty} \bar{\tau}^{n_{k}}\left(\theta_{0}\right)=\lim _{k \rightarrow-\infty} \bar{\tau}^{n_{k}}\left(\theta_{0}\right)$. Let $\left(r_{k}\right)$ be any enumeration of the 
rational numbers in $[-1,1]$ (any dense countable set would serve as well), and let $\bar{f}_{0}\left(\theta_{0}\right)=0, \bar{f}_{0}\left(\tau^{n 2 k-1}\left(\theta_{0}\right)\right)=0, \bar{f}_{0}\left(\tau^{n_{2 k}}\left(\theta_{0}\right)\right)=r_{k}$, and let $\bar{f}_{0}(\theta)$ be linear between successive pairs of points whose values have been defined above. In case $\theta_{0}$ is an endpoint of an interval $I_{n}$, first let $f(\theta)=0$ for $\theta \in I_{n}-\left\{\theta_{0}\right\}$. As before $\bar{f}(\theta)=2 A+\sum_{-\infty}^{\infty} \alpha_{n} \bar{f}_{n}(\theta), \theta \in \Theta$. Let

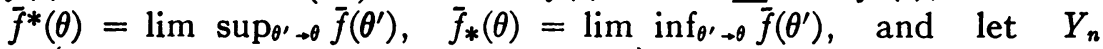
$=\left\{\left(\bar{\tau}^{n}\left(\theta_{0}\right), y\right) / \bar{f}_{*}\left(\bar{\tau}^{n}\left(\theta_{0}\right)\right) \leqq y \leqq \bar{f}^{*}\left(\bar{\tau}^{n}\left(\theta_{0}\right)\right)\right\}$, and let $Y=U_{-\infty}^{\infty} Y_{n}$. We show that $\bar{Y}$ is a minimal set under $\bar{\Psi}$. Let $\bar{F}(\theta)=\bar{f}(\theta)-\alpha_{0} \bar{f}_{0}(\theta)$. The saltus of $\alpha_{n} \bar{f}_{n}(\theta)$ at $\bar{\tau}^{n}\left(\theta_{0}\right)$ is $2 \alpha_{n}$. Since $\bar{F}^{*}\left(\theta_{0}\right)=\bar{F}_{*}\left(\theta_{0}\right), \theta_{0}$ is a point of continuity of $\bar{F}(\theta)$. Let $y$ be any number such that $\bar{f}_{*}\left(\theta_{0}\right) \leqq y \leqq f^{*}\left(\theta_{0}\right)$. There is a sequence $\left(\theta_{n}\right)$ such that $\theta_{n} \in \Theta$, and $\lim \bar{f}\left(\theta_{n}\right)=y, \lim \theta_{n}=\theta_{0}$. Let $w_{0}=\lim \alpha_{0} \bar{f}_{0}\left(\theta_{n}\right)=y-\bar{F}^{*}\left(\theta_{0}\right)$. Since $\left|w_{0}\right| \leqq \alpha_{0}$, there is a sequence of $\left\{\bar{\tau}^{n}\left(\theta_{0}\right)\right\}$, a subsequence of $\left(\bar{\tau}^{n_{2 k}}\left(\theta_{0}\right)\right)$, say $\left(\bar{\tau}^{n_{j}}\left(\theta_{0}\right)\right)$ such that $\lim \bar{f}_{0}\left(\bar{\tau}^{n_{j}}\left(\theta_{0}\right)\right)=w_{0}$. Thus, $\lim \bar{f}\left(\bar{\tau}^{n_{j}}\left(\theta_{0}\right)\right)=\lim \alpha_{0} \bar{f}_{0}\left(\bar{\tau}^{n_{j}}\left(\theta_{0}\right)\right)+\lim \bar{F}^{*}\left(\bar{\tau}^{n_{j}}\left(\theta_{0}\right)\right.$ $=w_{0}+\bar{F}^{*}\left(\theta_{0}\right)=y$.

We now conclude that any point of $Y_{0}$ is in the orbit-closure of any point of $Y_{0}$; since $Y_{n}=\bar{\Psi}^{n}\left(Y_{0}\right)$, seen immediately by taking limits, $Y$ is contained in the orbit-closure of any point of $Y_{0}$. Since the orbitclosure of a point is a minimal set if and only if the point is almost periodic (see [2, p. 31]), we have only to show that some point of $Y_{0}$ is almost periodic under $\bar{\Psi}$. Surely the candidate is $\left(\theta_{0}, \bar{F}^{*}\left(\theta_{0}\right)\right)$. The set $\left\{n_{2 k-1} / k \in \mathscr{g}\right\}$ is relatively dense in $\mathscr{g}$, since $\left\{n_{k} / k \in \mathfrak{g}\right\}$ is. But

$$
\lim _{k \rightarrow \pm \infty} \alpha_{0} \bar{f}_{0}\left(\bar{\tau}^{n_{2 k-1}}\left(\theta_{0}\right)\right)+\lim _{k} \bar{F}^{*}\left(\bar{\tau}^{n_{2 k-1}}\left(\theta_{0}\right)\right)=0+\bar{F}^{*}\left(\theta_{0}\right)
$$

so that

$$
\lim _{x \rightarrow \pm \infty} \bar{f}^{*}\left(\bar{\tau}^{n_{2 k-1}}\left(\theta_{0}\right)\right)=\bar{F}^{*}\left(\theta_{0}\right)=\lim _{k \rightarrow \pm \infty} \bar{f}_{*}\left(\bar{\tau}^{n_{2 k-1}}\left(\theta_{0}\right)\right) .
$$

Thus, from the definition of $\bar{\Psi}$,

$$
\lim \bar{\Psi}^{n_{22-1}}\left(\theta_{0}, \bar{F}^{*}\left(\theta_{0}\right)\right)=\left(\theta_{0}, F^{*}\left(\theta_{0}\right)\right)
$$

which establishes the almost periodicity of $\left(\theta_{0}, \bar{F}^{*}\left(\theta_{0}\right)\right)$.

It is clear that $\bar{Y}$, the above minimal set in $C(1)$, contains a bisequence $\left(Y_{n}, n \in \mathfrak{g}\right)$ of nondegenerate components as well as an uncountable collection of point components. If $Z_{n}$ is the homeomorph, under $\sigma$, of $Y_{n}$, the same holds for $\bar{Z}$ under $\bar{\Phi}$. Thus there is a homeomorphism of the plane onto itself which is orientation-preserving and whose collection of minimal sets contains one containing an infinite number of nondegenerate components. We draw attention also to the facts that there is one fixed point, the origin, and $\bar{\Phi}$ is not almost periodic on any of the invariant simple closed curves. 


\section{REFERENCES}

1. M. L. Cartwright, Some decomposition theorems for certain invariant continua and their minimal sets, Fund. Math. vol. 48 (1960) pp. 229-250.

2. W. H. Gottschalk and G. A. Hedlund, Topological dynamics, Amer. Math. Soc. Colloquium Publications, Vol. 36, 1955.

3. W. H. Gottschalk, Minimal sets: An introduction to topological dynamics, Bull. Amer. Math. Soc. vol. 64 (1958) pp. 336-351.

UNIVERSITY OF DELAWARE AND University of Cambridge

\section{THE KRONECKER PRODUCT OF GRAPHS}

\section{PAUL M. WEICHSEL}

Introduction. This note considers a graph product derived from the Kronecker product of matrices. Some indication of the geometrical nature of this product is given and a theorem stating necessary and sufficient conditions for a graph product to be connected is proved. The matrix analogue of the above result is also stated.

I. A convenient representation for a finite undirected graph [1] $G$ is an adjacency matrix. If the vertex set of $G$ is $\left\{p_{i}\right\}, i=1, \cdots, n$, then an adjacency matrix of $G$ is an $n \times n$ matrix $\left(a_{i j}\right)$ with $a_{i j}$ equal to the number of lines (paths of length one) joining $p_{i}$ to $p_{j}$. A given graph is then represented by an equivalence class of matrices $\bar{A}=\left\{P_{i} A P_{i}^{-1} \mid\right.$ for all permutation matrices $P_{i}$ of order equal to the order of $A\}$. Each element of $\bar{A}$ corresponds to a different ordering of the vertices of $G$. It is clear that for each such class of adjacency matrices there corresponds a unique class of isomorphic graphs.

From this point on "graph" will mean a finite undirected graph with no loops. Such a graph has an adjacency matrix $\left(a_{i j}\right)$ whose entries are non-negative integers such that $a_{i j}=a_{j i}$ and $a_{i i}=0$. We also use the following notation: $o(G)$ is the number of vertices of $G$ and is called the order of $G, p_{1} \rightarrow p_{k}$ is a chain in $G$ from vertex $p_{1}$ to vertex $p_{k}$, and $n\left(p_{1} \rightarrow p_{k}\right)$ is the number of lines (not necessarily distinct) in $p_{1} \rightarrow p_{k}$.

If $A$ and $B$ are two adjacency matrices and $A \circ B$ is some matrix product which is also an adjacency matrix then this matrix operation

Presented to the Society, September 1, 1961; received by the editors November 20, 1960. 